\title{
Reflecting Upon Different Perspectives on Specialized Advanced Mathematical Knowledge for Teaching
}

\author{
Miguel Ribeiro, Arne Jakobsen, Alessandro Ribeiro, \\ Nick H. Wasserman, José Carrillo, Miguel Montes and Ami Mamolo
}

Teachers' knowledge assumes a major role in practice and in students' learning and achievement. In particular, the construct of horizon knowledge or what can be termed specialized advanced mathematical knowledge for teaching (in order to capture the overall perspectives we are dealing with) has been the focus of attention from a variety of researchers with different foci. From this perspective, and aiming to deepen our understanding of such a construct, the aim of this working group was to discuss (and reflect upon) different theoretical perspectives, methodological approaches, and analytic methods used when focusing on such specialized advanced mathematical knowledge for teaching. In particular, we consider the activities of analyzing and conceptualizing situations where access and development of such teachers' knowledge is of primary importance.

Following work previously developed (in a DG at PME 34; Wasserman, Mamolo, Ribeiro, \& Jakobsen, 2014), this workshop aimed at continuing and deepening the discussions. We had three slots. In the first, a brief overview of the different perspectives of conceptualizing the specialized advanced knowledge for teaching was given by the proposed organizers, representing four different approaches and understandings of this construct. Afterwards, the participants were engaged in commenting, solving, reflecting on, and discussing two situations (one vignette and one episode) designed to access and develop teachers' advanced mathematical knowledge linked with the tasks of teaching. This discussion aimed at discussing the participants' interpretations of the different aspects of advanced mathematical knowledge involved that can (potentially) be explored having the provided situations as a starting point - and, in case of a need for changes in such situations, what would be the focus of such changes. A global discussion followed, aiming at both

\footnotetext{
M. Ribeiro ( $₫)$

State University of Campinas-UNICAMP, Campinas, Brazil

e-mail: cmribas78@gmail.com

(C) The Author(s) 2017

G. Kaiser (ed.), Proceedings of the 13th International Congress on Mathematical

Education, ICME-13 Monographs, DOI 10.1007/978-3-319-62597-3_135
} 
synthesizing and enhancing the participants' views and understanding of the construct at hand and fostering a deeper understanding of what such a construct comprises and the nature of the associated tasks for developing it (and its differences with other aspects of teachers' knowledge). Based on the different perspectives of the construct and the subsequent analyses of the two explored situations, we proposed to the WG participants the possibility of collaborating on papers/book chapters that would be an outcome of the group and would lead to a broader understanding of what comprises a construct of advanced mathematical knowledge for teaching as well as its potential implications for future research in this area.

\section{Reference}

Wasserman, N., Mamolo, A., Ribeiro, M., \& Jakobsen, A. (2014). Exploring horizons of knowledge for teaching. Discussion Group at PME 38, July 15-20, 2014, Vancouver, Canada.

Open Access Except where otherwise noted, this chapter is licensed under a Creative Commons Attribution 4.0 International License. To view a copy of this license, visit http://creativecommons. org/licenses/by/4.0/.

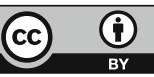

